\title{
PREVALENCIA DE ENFERMEDADES DE IMPACTO REPRODUCTIVO EN BOVINOS DE LAESTACIÓN EXPERIMENTAL DE TRÓPICO DEL CENTRO DE INVESTIGACIONES IVITA ${ }^{1}$
}

\author{
Hermelinda Rivera G. ${ }^{2,3}$, Alfredo Benito Z. ${ }^{2}$, Olger Ramos C. ${ }^{4}$ y Alberto Manchego S. ${ }^{2}$
}

\section{Abstract}

The prevalence of infectious agents associated with reproductive failures in Zebu and crossbred cattle $(n=268)$, older than six months of age, from the IVITA Research Center herd in Pucallpa, Peru, was determined. Serum samples were collected for the detection of antibodies against Brucella sp., Leptospira serovares: canicola, icterohemorrhagiae, pomona and hardjo, Bovine Viral Diarrhea Virus (BVDV), Bovine Herpes Virus Type 1 (BHV-1) and Neospora caninum by a commercial indirect ELISA, microglutination, viral neutralization and indirect immunofluorescence tests, respectively. No antibodies were detected againstBrucella sp., L. pomona and BVDV. The $46.3 \pm 6.0 \%$ of the animals had antibodies against BHV-1. The highest seroprevalence of BHV-1 $(63.2 \%)$ was found in cows older than 18 months. Antibody titres against BHV-1 varied from 2 till $>256$. The $52.2 \%$ of the animals had antibodies against Leptospira. The higher prevalence $(35 \%)$ corresponded to $L$. hardjo followed by $L$. canicola and $L$. icterohemorrhagiae (14.9\%). The titers of antibodies ranged from 1:100 to 1:400. Antibodies against $N$. caninum was found in $1.5 \%$ of the samples. These results indicate that Brucella sp. and BVDV are not prevalent in the herd. The eradication of $N$. caninum is feasible due to its low prevalence, but BHV-1 and Leptospira could be considered of sanitary risk for this herd.

Key words: bovine, tropics, abortogenic microorganisms, prevalence, antibodies

\section{Resumen}

\begin{abstract}
El objetivo del presente estudio fue determinar la prevalencia de agentes infecciosos con impacto reproductivo en bovinos de la Estación Experimental del Trópico del Centro de Investigaciones IVITA, en Pucallpa, Perú. Con este fin, se obtuvieron muestras de suero $(n=268)$ de bovinos cruzados y cebú, mayores de 6 meses de edad, para la detección de anticuerpos contra los virus de la diarrea viral bovina (VDVB) y herpes bovino1 (VHB-1), las bacterias Brucella sp. y Leptospira serovares: hardjo, pomona, canicola e icterohemorrhagiae y el protozoo Neospora caninum, mediante técnicas de neutraliza-
\end{abstract}

${ }^{1}$ Trabajo financiado por el Consejo Superior de Investigaciones de la UNMSM

${ }^{2}$ Laboratorio de Microbiología y Parasitología Veterinaria, FMV-UNMSM

${ }^{3}$ E-mail: hriverag@vet.unmsm.edu.pe

${ }^{4}$ Estación Experimental del Centro de Investigaciones IVITA-Pucallpa, FMV-UNMSM 
ción viral, ELISA indirecta, microaglutinación e inmunofluorescencia indirecta, respectivamente. No se detectaron anticuerpos contra el VDVB ni Brucella sp. en los animales muestreados. El $46.3 \pm 6.0 \%$ de los animales presentaron anticuerpos contra el VHB-1, correspondiendo el $63.2 \%$ a animales adultos. Los títulos de anticuerpos contra el VHB1 variaron entre $2 \mathrm{a}>256$. El 52.2\% de los animales tuvieron anticuerpos contraLeptospira. La $L$. hardjo tuvo mayor prevalencia $(35 \%)$ seguido por la $L$. canicola y $L$. icterohemorrhagiae (14.9\%). No se detectaron seroreactores contra la L. pomona. Los títulos de anticuerpos leptospirales estuvieron en el rango de 100 a 400. El 1.5\% de los animales fueron reactores a $N$. caninum. Estos resultados indican que la Brucella sp. y el VDVB no son prevalentes en el hato en estudio. La baja prevalencia de $N$. caninum en los animales hace posible su erradicación del hato, mientras que el VHB-1 y la Leptospira constituyen agentes infecciosos de riesgos sanitarios.

Palabras clave: bovino, trópico, microorganismos abortogénicos, prevalencia, anticuerpos

\section{INTRODUCCIÓN}

Los problemas reproductivos, caracterizados por infertilidad, muerte embrionaria, abortos, malformaciones congénitas, nonatos y nacidos débiles son prevalentes en el ganado bovino ocasionando serias pérdidas económicas (Houe, 1995; McGowan y Kirkland, 1995). Los problemas reproductivos en el ganado bovino tienen múltiples etiologías; y los agentes infecciosos, como los virus de la diarrea viral bovina (VDVB) y el herpes bovino 1 (VHB-1), agente causal de la rinotraqueítis infecciosa bovina, el parásito Neospora canimun y las bacterias Brucella sp., y Leptospira sp. están ampliamente distribuidos en la población bovina (Brownlie et al., 1998; Wounda et al., 1999).

Estudios realizados en bovinos lecheros, principalmente en los valles de Lima, Arequipa y el Mantaro, han determinado que los agentes DVB y $N$. caninum son los principales causantes de abortos (Rivera, 2001). Abortos causados por Brucella sp. y Leptospira sp. se reportan, además, en el ganado lechero de Lima, Arequipa y otras áreas del país, aunque en forma esporádica (Cárdenas y Rivera, 2001). Por otro lado, el VDVB, conjuntamente con el VHB-1 y otros virus neumotrópicos, constituyen agentes predisponentes a infecciones secundarias del tracto respiratorio, especialmente en los primeros meses de vida del animal (Rivera et al., 1994; Stahl et al., 2002).

Actualmente, la ganadería lechera se viene desarrollando en algunas zonas de la Amazonía peruana, como el Alto Mayo en San Martín y Pucallpa en Ucayali; sin embargo, existe escasa información sobre la prevalencia de las enfermedades antes mencionadas que afectan la vida reproductiva del animal. La Estación Experimental (EE) del Centro de Investigación IVITA de la Facultad de Medicina Veterinaria (FMV), Universidad Nacional Mayor de San Marcos (UNMSM), ubicado en Pucallpa, Amazonía Baja, posee un hato cerrado con aproximadamente 500 bovinos cebú y cruzados cebú $\mathrm{x}$ europeo, criados en forma extensiva, con un gran potencial como centro de recría; por lo que el objetivo del presente estudio fue determinar la seroprevalencia de las enfermedades de impacto reproductivo en ese centro pecuario, con el propósito de que los datos puedan ser utilizados para el mejoramiento de su estado sanitario.

\section{Materiales y Métodos}

Se colectaron muestras de sangre, en el 2002, de todos los bovinos mayores a 6 meses de edad $(n=268)$ del hato de la EE 
Cuadro 1. Detección de anticuerpos contra el virus herpes bovino 1 (VHB-1) y Neospora caninum en bovinos de la Estación Experimental del Centro de investigación IVITA, Pucallpa. 2002

\begin{tabular}{|c|c|c|c|c|c|}
\hline \multirow{3}{*}{ Grupo } & \multirow{3}{*}{$\begin{array}{c}\mathrm{N}^{\circ} \mathrm{de} \\
\text { animales }\end{array}$} & \multicolumn{4}{|c|}{ Anticuerpos contra } \\
\hline & & \multicolumn{2}{|c|}{ VHB-1 } & \multicolumn{2}{|c|}{ N. caninum } \\
\hline & & $\mathrm{n}$ & $\%$ & $\mathrm{n}$ & $\%$ \\
\hline Vacas & 193 & 122 & 63.2 & 4 & 2.1 \\
\hline Vaquillas & 46 & 1 & 2.2 & 0 & 0 \\
\hline Toretes & 29 & 1 & 3.4 & 0 & 0 \\
\hline Total & 268 & 124 & $46.3 ? 6.0^{1}$ & 4 & 1.5 \\
\hline
\end{tabular}

${ }^{1}$ Intervalo de confianza del $95 \%$

del IVITA-Pucallpa. Las muestras fueron centrifugadas para la obtención del suero en el laboratorio de la EE del IVITA, Pucallpa, y almacenadas en viales para su transporte a Lima.

En el Laboratorio de Virología de la FMV-UNMSM se realizó la detección de anticuerpos contra los diversos agentes en estudio. Se utilizó la técnica de neutralización viral para el caso del VDVB y VHB-1; inmunofluorescencia indirecta para $N$. caninum, ELISA indirecta para Brucella sp., y microaglutinación empleando como antígeno vivo los serovares de Leptospira: pomona, canicola, icterohemorrhagiae y hardjo, según los protocolos utilizados en el laboratorio.

\section{Resultados y Discusión}

Ninguno de los animales muestreados presentó anticuerpos contra Brucella sp. ni contra VDVB, indicando que los animales no tuvieron contacto con estos agentes infecciosos. En estudios preliminares sobre la prevalencia de la brucelosis en el ganado bovino efectuados en Pucallpa, el Alto Mayo y Tarapoto se determinaron valores inferiores al $1 \%$, en tanto que datos obtenidos en las décadas del '70 y '80 en la zona de
Pucallpa señalaban entre el 2 al 5\% (A. Trigueros, comunicación personal; Informe IAEA/FAO, 1999). La reducción de la prevalencia de la brucelosis se debe posiblemente al esfuerzo compartido entre los ganaderos organizados y las autoridades sanitarias quienes cumplen y hacen cumplir el Reglamento para el Control y Erradicación de la Brucelosis Bovina. Sin embargo, se tiene reportes de la introducción de la brucelosis a un área libre con bovinos de otra procedencia, como el brote ocurrido en Madre de Dios (Cárdenas y Rivera, 2001). La presentación esporádica de la brucelosis en el trópico y otras áreas del país hace que esta enfermedad continúe siendo una amenaza para la vida reproductiva del animal y para la salud pública, por ser también una importante zoonosis. La ausencia de la infección en el hato del IVITA no significa su ausencia en Pucallpa, por lo que debe evitarse el contacto de los animales con otros bovinos, caprinos y porcinos.

El VDVB tiene una amplia distribución en los bovinos del país, con prevalencias que van de 0 a más del $90 \%$ (H. Rivera, comunicación personal); de allí que la ausencia de este patógeno en el hato de la EE del IVITA sugiere que no se han introducido bovinos infectados o que ha habido poco contacto de estos animales con otros rumiantes infecta- 


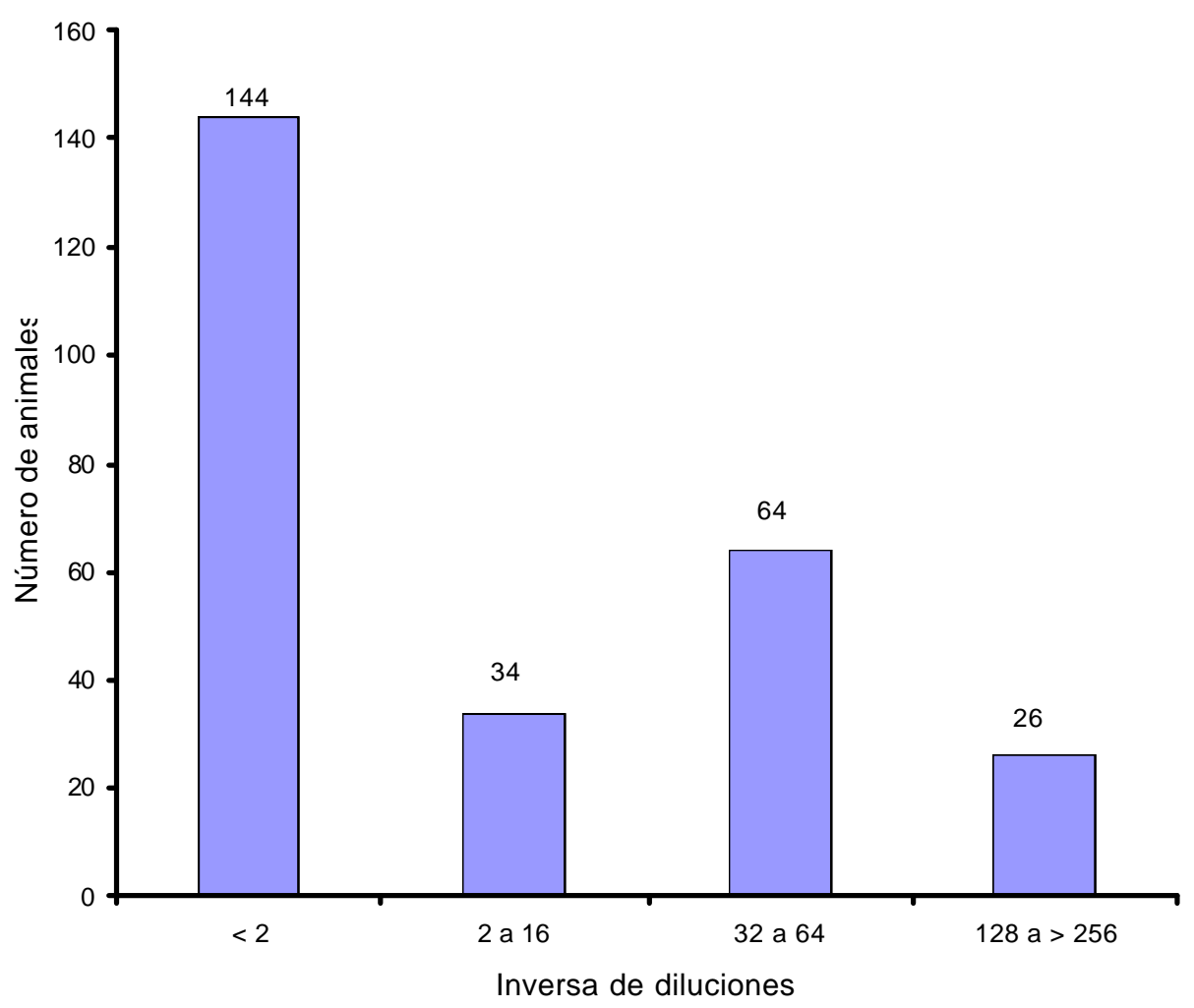

Figura 1. Distribución de los títulos de anticuerpos contra el VHB-1 en bovinos de la EE del IVITA, Pucallpa, 2002.

dos, que es el modo usual de introducción del VDVB a un hato o una región. En poblaciones de animales susceptibles, el virus se transmite con eficiencia, sobre todo en hatos de crianza intensiva o durante la concentración del ganado para actividades sanitarias en caso de la crianza extensiva (Houe, 1995).

No existen datos de prevalencia de la DVB en bovinos del trópico y menos aún de Pucallpa. Si en algún momento la infección fue introducida a la zona, es posible que se haya autolimitado debido a la reducida población de animales y al tipo de crianza, minimizándose su transmisión. La ausencia del virus en el hato concuerda con la baja frecuencia de abortos o pérdidas embrionarias que se reportan (O. Ramos, comunicación personal) pero también constituye un riesgo de ingreso del agente si no se mantiene el hato cerrado.
El $46.3 \%$ de los animales presentaron anticuerpos contra el VHB-1, correspondiendo el $63.2 \%$ a hembras mayores de 18 meses de edad (Cuadro 1). Estudios de seroprevalencia realizados en algunas áreas del país indican que las infecciones en animales adultos con el VHB-1 son de tipo subclínico (Zacarías et al., 2002, Sánchez et $a l ., 2003)$, pero el virus puede permanecer latente en el ganglio del nervio trigémino o del nervio sacro de donde pude reactivarse ante cualquier estrés, manteniéndose de este modo en el medio. Estos animales son fuentes de infección para los animales jóvenes, afectando severamente el tracto respiratorio. El virus se comporta como un agente primario en el complejo respiratorio. En países con marcadas variaciones climáticas como los Estados Unidos, los animales adultos y jóvenes pueden presentar la forma aguda caracterizada principalmente por una severa 
rinotraqueitis que puede progresar a una neumonía, y donde muchos de los animales que se recuperan quedan portadores del virus (Banks, 1999).

Los títulos de anticuerpos contra el VHB-1 variaron entre $2 \mathrm{a}>256$ (Fig. 1), indicando una activa presencia del virus en los animales adultos. Los títulos de anticuerpos en un rango de 32 y 64 en el $23 \%$ de los animales seroreactores sugiere la predominancia de infecciones subclínicas que generalmente presentan los animales adultos $(\mathrm{H}$. Rivera, datos no publicados), pero el hallazgo de títulos entre 128 a $>256$ en el $9.7 \%$ de los animales (Fig.1) indican una activa presencia del virus en estos animales o continuas reactivaciones como consecuencia de factores estresantes, tales como falta de alimento, deficiencias nutricionales o presencia de superinfecciones con otros agentes, como por ejemplo, el parásito Dictyocaulus viviparus (Wentink et al., 1993; Lorraine et al., 1994). A pesar de los altos títulos de anticuerpos, no se reportaron signos clínicos ni un incremento de los abortos en el hato durante el año en estudio confirmando el carácter subclínico de la infección (Wentink et al., 1993; Lorraine et al., 1994).

La historia clínica del hato indica que los problemas respiratorios no son frecuentes entre los animales jóvenes, lo cual es concordante con la baja prevalencia del VHV-1 en las vaquillas y toretes, sugieriendo que el sistema de manejo de tipo extensivo contribuye a disminuir la transmisión viral entre los animales susceptibles, manteniéndose posiblemente la infección en el mismo animal.

La neosporosis no está, al parecer, muy difundida en el hato, pues sólo el 1.5\% de los bovinos adultos presentaron anticuerpos contra el protozoo $N$. caninum (Cuadro 1). La escasa difusión del $N$. caninum en el hato en estudio podría ser debido a la nula introducción de animales positivos o a la ausencia de perros infectados. En comparación, en un estudio realizado en bovinos lecheros de la provincia de
Chachapoyas, Amazonas (zona de selva alta), se menciona una prevalencia del $40 \%$ (Quevedo et al., 2003). Esta diferencia pudo deberse a que allí hubo introducción de animales, principalmente de Cajamarca, donde se reporta una prevalencia de $N$. caninum superior al 50\% (Cabrera et al., 2000). La baja prevalencia del parásito en la EE IVITA constituye una ventaja para la inmediata erradicación de los animales reactores del hato, y una señal de alerta para reforzar la bioseguridad, no introduciendo animales sin un previo descarte de este parásito.

La leptospira tuvo una prevalencia de $52.2 \%$ y una frecuencia mayor en adultos. El serovar hardjo tuvo la mayor prevalencia (35\%), seguido por los serovares canicola e icterohemorrhagiae (14.9\%), en tanto que no se detectaron anticuerpos contra el serovar pomona. Anticuerpos contra los serovares canicola e icterohemorrhagiae fueron detectados en el $29.3 \%$ de los animales reactores y en el $6.4 \%$ contra los tres serovares en forma simultánea, evidenciando infecciones mixtas. Esto es factible ya que los anticuerpos son serovar específico. Los anticuerpos tuvieron un título entre 100 a 400 sugiriendo infecciones no agudas, ya que durante una infección aguda se producen títulos superiores a 10,000. La asociación de abortos con Leptospira se establece siempre que los títulos durante el aborto sean muy altos.

La leptospirosis en animales no gestantes son usualmente de tipo subclínico, pero desarrollan anticuerpos poco después de la leptospiremia. Bolin y Alt (1999) en Estados Unidos mencionan una prevalencia de $29 \%$ para el serovar hardjo y de $19 \%$ para icterohemorrhagiae. El serovar hardjo es el más importante en la población bovina por su carácter endémico. Si un animal es infectado con este serovar y no es eliminado por los anticuerpos, la leptospira puede establecerse en los riñones o en el tracto genital ocasionado fallas reproductivas (Bolin y Alt, 1999; Langoni et al., 1999). Todos los serovares de Leptospira patógena incluyendo el serovar pomona pueden ocasionar fallas 
reproductivas, como el aborto en el ganado bovino; sin embargo, la ausencia del serovar pomona en los animales del hato llama la atención. Posiblemente este serovar tiene baja prevalencia en zona de Pucallpa o no hubieron animales reservorios de mantenimiento de Leptospira en la zona durante el tiempo de colección de las muestras, ya que un similar estudio realizado en sajinos (Tayassu tayacu) de algunos zoocriaderos de Pucallpa no se encontraron anticuerpos contra el serovar pomona (Mendoza, 2004).

La historia clínica del hato en estudio indica que la incidencia de abortos es baja, sugiriendo la ausencia o escasa participación de los agentes abortogénicos como Brucella sp., Leptospira sp., DVB o N. caninum. La presencia del VHB-1 podría ocasionar problemas respiratorios en los animales jóvenes.

El presente estudio ha permitido conocer que enfermedades de impacto económico como la brucelosis, la diarrea viral bovina y la neosporosis están ausentes o tienen baja prevalencia en el hato de la EE del IVITA-Pucallpa; pero otras como leptospirosis y la rinotraqueitis infecciosa (VHB-1) están presentes constituyendo un riesgo sanitario, por lo que se recomienda maximizar las medidas de bioseguridad, evitando el ingreso de animales sin control sanitario y realizar una permanente vigilancia epidemiológica de estas enfermedades.

\section{Literatura Citada}

1. Banks, M. 1999. Living with IBR. Holstein J. 3: 84-87.

2. Bolín, C.A.; D.P. Alt. 1999. Clinical signs, diagnosis and prevention of bovine leptospirosis. Bovine Practitioner 33: 50-55.

3. Brownlie, J.; L.B. Hooper; I. Thompson; M.E. Collins. 1998. Maternal recognition of foetal infection with bovine virus diarrhea virus - the bovine pestivirus. Clin. Diagn. Virol. 10: 141150.
4. Cabrera, M.; P. Ortiz; J. Claxton; D. Williams; A. Trees. 2000. Evidencia serológica de infección por Neospora caninum en ganado vacuno en Perú. Libro de Resúmenes IV Congreso Peruano de Parasitología. Lima. p 212.

5. Cárdenas, J.; H. Rivera. 2001. Brucella abortus en bovinos de la provincia de Tambopata, Madre de Dios. Rev. Acad. peru. cienc. vet. 2: 6-10.

6. Houe, H. 1995. Epidemiology of bovine viral diarrhea virus. Vet. Clin. North Am.: Food Animal Practice 11: 521-547.

7. Informe Anual del Proyecto de Cooperación Técnica PER/5/023. 1999. Agencia Internacional de Energía Atómica (IAEA/FAO). p 15-18.

8. Langoni, H.; L. Carlos de Souza; A. Vieira da Silva; M.C. Luvizotto; A.C. Paes; S.B. Lucheis. 1999. Incidence of leptospiral abortion in Brasilian dairy cattle. Prev. Vet. Med. 40: 271-275.

9. Lorraine, A.B.; R.A. Fenton; V. Misra; D.M. Haines. 1994. Fetal, generalized bovine herpesvirus type 1 infection associated with a modified-live infectious bovine rhinotracheitis, parainfluenza-3 vaccine administered to neonatal calves. Can. Vet. J. 35: 223-227.

10. McGowan, M.R.; P.D. Kirkland. 1995. Early reproductive loss due to bovine pestivirus infection. Br. Vet. J. 151: 263270.

11. Mendoza, A.P. 2004. Presencia de anticuerpos contra Leptospira interrogans en sajinos (Tayassu tajacu) provenientes de zoocriaderos de la Amazonía Peruana. Tesis de Médico Veterinario. Facultad de Medicina Veterinaria, Univ. Nacional Mayor de San Marcos. Lima $80 \mathrm{p}$.

12. Quevedo, J.M; A. Chávez; H. Rivera; E. Casas; E. Serrano. 2003. Neosporosis en bovinos lecheros en dos distritos de la provincia de Chachapoyas. Rev. Inv. Vet. Perú 14: 33-37.

13. Rivera, H. 2001. Etiología infecciosa del aborto bovino. Rev. Inv. Vet. Perú, Supl. 1: 95-99. 
14. Rivera, H.; A. Manchego; N. Sandoval; E. Flores. 1994. Complejo respiratorio bovino en terneros del valle de Lima. Rev. Inv. Pec. IVITA (Perú) 7: 35-38.

15. Stahl, K.; H. Rivera; I. Vagsholm; J. Moreno-López. 2002. Bulk milk testing for antibody seroprevalence to BVDV and BHV-1 in a rural region of Peru. Prev. Vet. Med. 56: 193-202.

16. Sánchez, G.; A. Benito; H. Rivera. 2003. Seroprevalencia del virus de la rinotraqueitis infecciosa en ganado lechero lechero del valle de Lima. Rev. Inv. Vet. Perú 14: 54-60.
17. Wentink, G.H.; J.T. van Oirschot; J. Verhoeff. 1993. Risk of infection with bovine herpes virus 1 (BHV-1): A review. Vet. Q. 15: 30-33.

18. Wounda, W.; Th. Dijkstra; A.M.H. Kramer; C. Maanen; J.M. Brinkhof. 1999. Seroepidemiological evidence for a relationship between Neospora caninum infections in dogs and cattle. J. Parasitol. 29: 1677-1682.

19. Zacarías, E.; A. Benito; H. Rivera. 2002. Seroprevalencia del virus de la rinotraqueitis infecciosa en bovinos criollos de Parinacochas, Ayacuacho. Rev. Inv. Vet. Perú 13: 61-65. 